Research Paper

\title{
Salvianolic acid B, a novel autophagy inducer, exerts antitumor activity as a single agent in colorectal cancer cells
}

\author{
Zhao Jing ${ }^{1,2, *}$, Weiqiang Fei ${ }^{2, *}$, Jichun Zhou ${ }^{3, *}$, Lumin Zhang ${ }^{2}$, Liuxi Chen ${ }^{1}$, Xiaomin \\ Zhang ${ }^{1}$, Xiao Liang ${ }^{4}$, Jiansheng $X_{i e}{ }^{2}$, Yong Fang ${ }^{1,2}$, Xinbing Sui ${ }^{1,2}$, Weidong Han ${ }^{1,2}$, \\ Hongming Pan ${ }^{1,2}$ \\ ${ }^{1}$ Department of Medical Oncology, Sir Run Run Shaw Hospital, College of Medicine, Zhejiang University, Hangzhou, Zhejiang, \\ China \\ ${ }^{2}$ Biomedical Research Center and Key Laboratory of Biotherapy of Zhejiang Province, College of Medicine, Zhejiang University, \\ Hangzhou, Zhejiang, China \\ ${ }^{3}$ Department of Surgical Oncology, Sir Run Run Shaw Hospital, College of Medicine, Zhejiang University, Hangzhou, Zhejiang, \\ China \\ ${ }^{4}$ Department of General Surgery, Sir Run Run Shaw Hospital, College of Medicine, Zhejiang University, Hangzhou, Zhejiang, \\ China \\ *These authors contributed equally to this work \\ Correspondence to: Xinbing Sui, email: hzzju@aliyun.com \\ Weidong Han, email: hanwd@126.com \\ Hongming Pan, email: drpanhm@aliyun.com
}

Keywords: salvianolic acid B, natural compound, autophagy, cell death, colorectal cancer

Received: April 25, 2016 Accepted: August 13, $2016 \quad$ Published: August 19, 2016

\section{ABSTRACT}

Salvianolic Acid B (Sal B), an active compound extracted from the Chinese herb Salvia miltiorrhiza, is attracting more and more attention due to its biological activities, including antioxidant, anticoagulant and antitumor effects. However, autophagy induction in cancer cells by Sal B has never been recognized. In this study, we demonstrated that Sal B induced cell death and triggered autophagy in HCT116 and HT29 cells in a dose-dependent manner. Specific inhibition of autophagy by 3-MA or shRNA targeting Atg5 rescued Sal B-induced cell death in vitro and in vivo, suggesting that Sal B-induced autophagy may play a pro-death role and contribute to the cell death of colorectal cancer cell lines. Furthermore, AKT/mTOR signaling pathway was demonstrated to be a critical mediator in regulating Sal B-induced cell death. Overexpression of AKT by the transfection with AKT plasmid or pretreatment with insulin decreased Sal B-induced autophagy and cell death. Inversely, inhibition of AKT by LY294002 treatment markedly enhanced Sal B-induced autophagy and cell death. Taken together, our results demonstrate, for the first time, that Sal B is a novel autophagy inducer and exerts its antitumor activity as a single agent in colorectal cancer cells through the suppression of AKT/mTOR pathway.

\section{INTRODUCTION}

Colorectal cancer (CRC) is still the third most common cancer and the third leading cause of cancerrelated death around the world, although considerable progress has been made in the treatment of CRC over the past years [1]. Currently, the initial chemotherapy with 5 -fluorouracil (5-FU) and its derivatives is effective in most CRC patients, the relapse rate remains high due to increasing resistance to chemotherapeutic agents $[2,3]$.
Therefore, identifying novel compounds and overcoming chemoresistance will be a key issue for developing more effective individualized therapeutic strategies for the treatment of CRC patients.

Macroautophagy (hereafter referred to as autophagy) is an evolutionarily conserved catabolic process by which damaged or dysfunctional cellular contents are delivered to lysosomes for bulk degradation [4]. Autophagy is regulated by various signaling pathways, and AMPactivated protein kinase (AMPK) and mammalian target 
of rapamycin (mTOR) signaling pathways have emerged as the central checkpoints in the regulation of autophagy [5]. The role of autophagy in regulating cancer cell fate remains controversial. Autophagy itself fulfils a dual role, having pro-survival and pro-death properties depending on tumor types and treatment characteristic [6]. In response to anticancer treatments, autophagy can be activated as a protective mechanism to mediate the acquired resistance to anticancer therapies. Paradoxically, on the other hand, autophagy may also function as a death executioner to induce autophagic cell death, a distinct form of cell death in contrast to type I programmed cell death or apoptosis [7-9].

In the past few years, natural products with anticancer activity have gained more and more attention due to their favorable safety and efficacy profiles. Salvia miltiorrhiza (Danshen), a well-known traditional Chinese medical herb, has been widely and successfully used for treating cardio- and cerebral vascular diseases, such as angina pectoris, myocardial infarction (MI) and stroke [10]. Salvianolic Acid B (Sal B), one of the major watersoluble compounds of Danshen, is the most abundant and bioactive component of the salvianolic acids in Danshen [10]. Previous studies have shown that Sal B possesses multiple desirable potentials, including anti-oxidation, anti-inflammation, anti-coagulation and indirect regulation of immune function [11-13]. Recent studies indicate that Sal B also exerts anticancer effects in several cancer cell lines including glioma, hepatoma, oral squamous carcinoma, as well as head and neck squamous cell cancers [14-17]. However, the effect of Sal B on autophagy and the survival of CRC cells has never been reported.

In the present study, we investigated the effect of Sal B on CRC cells. We demonstrated, for the first time, that Sal B was a novel autophagy inducer, with significant antitumor efficacy as a single agent by inducing autophagic cell death in CRC cells. Furthermore, we showed that AKT inhibition is a key determinant for Sal B-mediated autophagic cell death. To the best of our knowledge, this is the first research to demonstrate that Sal B induces autophagic cell death through the AKT-mTOR signaling in human CRC cells. Our results suggest that Sal B may be an attractive therapeutic strategy for the treatment of colorectal cancer.

\section{RESULTS}

\section{Sal B induces cell death and inhibits cell proliferation in CRC cell lines}

In order to examine whether Sal B (Figure 1A) affects human colorectal cancer cell growth, we first investigated the effect of Sal B on cell viability in HCT116 and HT29 cells. After treatment with different concentrations of Sal B for 24 and 48 h, Sal B significantly inhibited the growth of CRC cells in a dose- and timedependent manner (Figure 1B and 1C). Next, we used various concentrations of $\mathrm{Sal} \mathrm{B}$ in the treatment of HCT116 and HT29 cells for $24 \mathrm{~h}$ in subsequent experiments. Light microscopy showed that the viability of HCT116 and HT29 cells treated with Sal B was significantly lower than that of controls (Figure 1D), with more detached and shrunken cells appearing. To determine whether Sal B inhibits anchorage-independent growth, we performed colony formation assays through monolayer culture. In agreement with MTT viability assay results, Sal B remarkably decreased the number and the size of the colonies (Figure 1E). These results suggest that Sal B possesses growth-inhibitory potential in CRC cells as a single agent.

\section{Sal B triggers autophagy in CRC cell lines}

To investigated whether autophagy occurred in Sal B-treated cells, we examined the effect of Sal B on autophagy. After HCT116 and HT29 cells were treated with Sal B for $24 \mathrm{~h}$, we performed fluorescence assays for LC3B to validate the effects of Sal B on autophagy. As a result, specific punctate distribution of endogenous LC3-II was observed in Sal B-treated cells and the percentage of FITC-LC3 positive cells with punctate staining significantly increased in Sal B-treated cells, compared with their controls (Figure 2A). In addition, treatment of Sal B to stable CRC cell lines expressing GFP-tagged LC3 resulted in marked accumulation of green fluorescent dots than untreated controls, suggesting induction of autophagy (Figure 2B). Sal B-induced autophagic flux was further investigated in the presence and absence of autophagosome- lysosome fusion inhibitors, bafilomycin A1 (BafA1). HCT116 and HT29 cells were preincubated with $100 \mathrm{nM}$ BafA1 for $2 \mathrm{~h}$ and then treated with Sal B for $24 \mathrm{~h}$. As a result, enhanced accumulation of LC3 puncta was found after $24 \mathrm{~h}$ treatment of Sal B in cells pre-incubated with BafA1 (Figure 2B). We next performed western blotting analysis to detect cleaved LC3-II and found that a significantly increased LC3-II/I ratio was shown in HCT116 and HT29 cells treated with Sal B for $24 \mathrm{~h}$ (Figure 2C). At last, transmission electron microscopy was used to further confirm the morphological changes in Sal B-treated cells. As shown in Figure 2D, most of the HCT116 and HT29 cells with Sal B treatment displayed an extensive accumulation of double or multimembraned structures with a broad range of morphologies, indicating the formation of autophagosomes. These results suggest that aberrant autophagosome accumulation is involved in Sal B-treated cells.

\section{Sal B-induced cell death is partially apoptotic}

Next, we investigated the role of apoptosis in Sal B-induced cell death. Western blotting analysis showed that the cleaved caspase-9, caspase-3 and poly (ADPribose) polymerase (PARP) were increased in HCT116 
and HT29 cells treated with Sal B for 24 h (Figure 3A), indicating that apoptosis may be involved in Sal B-induced cell death. To further elucidate whether caspase activation is required for Sal B-induced cell death, we exposed cells to a pan caspase inhibitor Z-VAD-FMK. As shown in Figure $3 \mathrm{~B}$, the addition of Z-VAD-FMK $(25 \mu \mathrm{M})$ markedly suppressed the exoression of cleaved PARP1 and active caspase-9 induced by Sal B (Figure 3B). However, Sal $\mathrm{B}$-induced cell death was only modestly rescued in all the treated CRC cell lines (Figure 3C), which was confirmed by an Annexin V-FITC dual staining assay followed by flow cytometry. Taken together, our data demonstrate that Sal B induced cell death in a partially apoptotic manner.

\section{Autophagy contributes to sal B-induced cell death}

To assess whether autophagy contributed to Sal B-induced cell death in HCT116 and HT29 cells, we first treated these cells with the autophagy inhibitor 3-methyladenine (3-MA). 3-MA, a class III phosphatidylinositol 3-kinase (PtdIns3K) inhibitor, can block autophagosome formation. The combination of Sal B with 3-MA (10 mM) significantly decreased LC3-II/I ratio and the expression of Atg5 (Figure 4A), accompanying with reduced the accumulation of GFPLC3B puncta (Figure 4B). Importantly, autophagy inhibition by 3-MA rescued both HCT116 and HT29 cells from Sal B-induced cell death, which was demonstrated by flow cytometric analysis (Figure 4C).

To further explore the link between the inhibition of autophagy and Sal B-induced cell death, we silenced the expression of Atg 5, a key regulator of autophagy, with shRNA in HCT116 and HT29 cells. As shown in Figure 4D, the mRNA level of Atg5 was significantly downregulated, together with reduced the accumulation of GFP-LC3B puncta and decreased protein expression level of Atg5 (Figure 4B and 4E). To obtain objective quantification of cell death, we performed an Annexin V-FITC dual staining assay followed by flow cytometry. In agreement with the results from 3-MA, pretreatment with shAtg5 resulted in inhibiting Sal B-induced cell death (Figure 4F). In conclusion, induction of autophagy promotes the cell death of HCT116 and HT29 cells in response to $\mathrm{Sal} \mathrm{B}$, and inhibition of autophagy counteracts the cytotoxic effect of Sal B in these cells.

\section{AKT pathway is a critical mediator in regulating sal B-induced cell death}

Previous studies have shown that Akt/mTOR axis plays an important role in the inhibition of autophagy [18, 19]. Therefore, we investigated whether the Akt/mTOR pathway was involved in Sal B-induced autophagic
A<smiles></smiles>

B

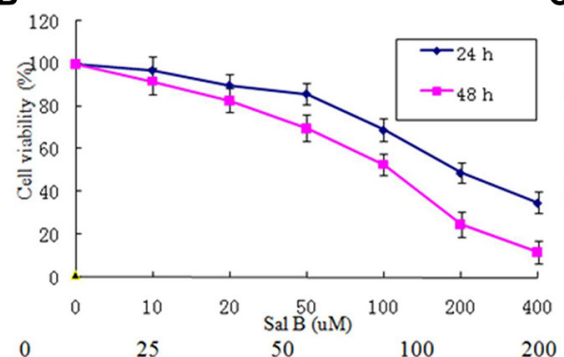

C

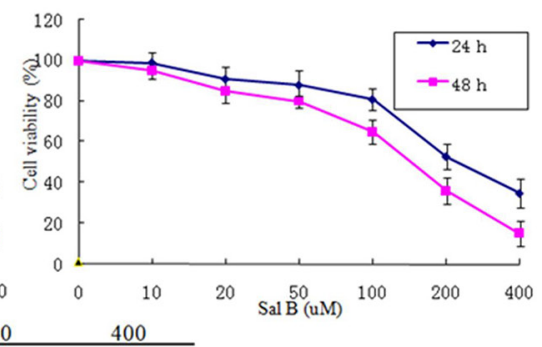

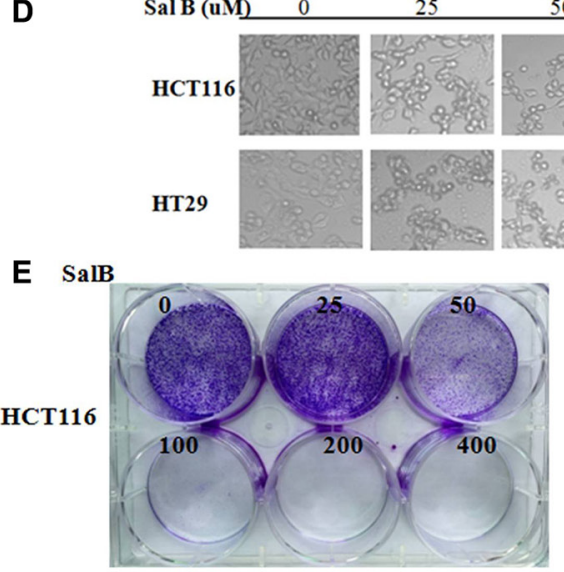
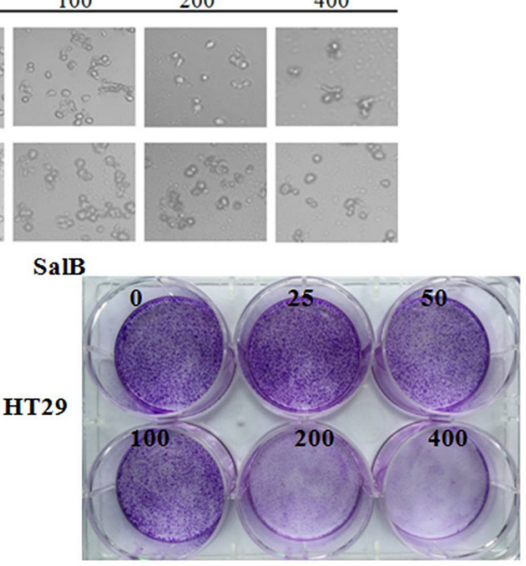

Figure 1: The effect of sal B on cell viability and proliferation in CRC cell lines. (A) The chemical structures of Sal B. (B) The cell viability of HCT116 cells was measured via the MTT assay after Sal B treatment. The experiments were performed in triplicate. (C) The cell viability of HT29 cells was measured via the MTT assay after Sal B treatment. The experiments were performed in triplicate. (D) Representative cell morphological changes are detected by light microscopy; characteristic morphological features of cell death were observed, including detachment and cell shrinkage. (E) Representative colony formation assay by monolayer culture. 
cell death in HCT116 and HT29 cells. Western blotting analysis showed that the phosphorylated levels of AKT, mTOR and p70 ribosomal protein S6 kinase (p70S6K) were significantly decreased but phosphorylated UNC-51Like kinase 1 (ULK1) was enhanced in Sal B-treated cells. This result indicates a potent inhibitory effect of Sal B on Akt/mTOR signaling (Figure 5A).

To further identify the role of AKT in Sal B-induced autophagy, HCT116 and HT29 cells were transiently transfected with pcDNA3-CA-AKT plasmid. As shown in Figure $5 \mathrm{~B}$ and $5 \mathrm{C}$, overexpression of AKT significantly reduced Sal B-induced autophagy. Importantly, enforced expression of AKT remarkably attenuated the cytotoxicity of Sal B on HCT116 and HT29 cells (Figure 5D). Moreover, pretreatment with insulin, an activator of $\mathrm{PI} 3 \mathrm{~K} / \mathrm{AKT} / \mathrm{mTOR}$ signaling pathway, significantly enhanced the phosphorylation of Akt but decreased Sal B-induced autophagy and cell death (Figure 5E and 5F). Inversely, pretreatment with LY294002, an inhibitor of $\mathrm{PI} 3 \mathrm{~K} / \mathrm{AKT} / \mathrm{mTOR}$ signaling pathway, markedly suppressed the phosphorylation of Akt but enhanced Sal
B-induced autophagy and cell death (Figure 5G and 5H). Taken together, these data demonstrate that AKT/ mTOR signaling is a critical mediator of Sal B-induced autophagic cell death, and AKT inhibition augments Sal B-induced cell death through inducing autophagy.

\section{Sal B exerts autophagy induction and antitumor efficacy in vivo}

To further determine the therapeutic potential of Sal B in vivo, we then injected BALB/c nude mice subcutaneously with HCT116 cells. The mice with tumor xenografts reaching $100 \mathrm{~mm}^{3}$ were randomly divided in to 4 experimental groups: control group, 3-MA group $(20 \mathrm{mg} / \mathrm{kg}$, intraperitoneal injection, every 4 days, for 28 days), Sal B group ( $80 \mathrm{mg} / \mathrm{kg}$, intraperitoneal injection, once daily, for 28 days) and Sal B plus 3-MA group. As shown in Figure 6A and 6B, 3-MA alone had no significant effect on the growth of the tumors and Sal B alone displayed a strong antitumor activity. Consistent with the in vitro results, a combination with the autophagy inhibitor

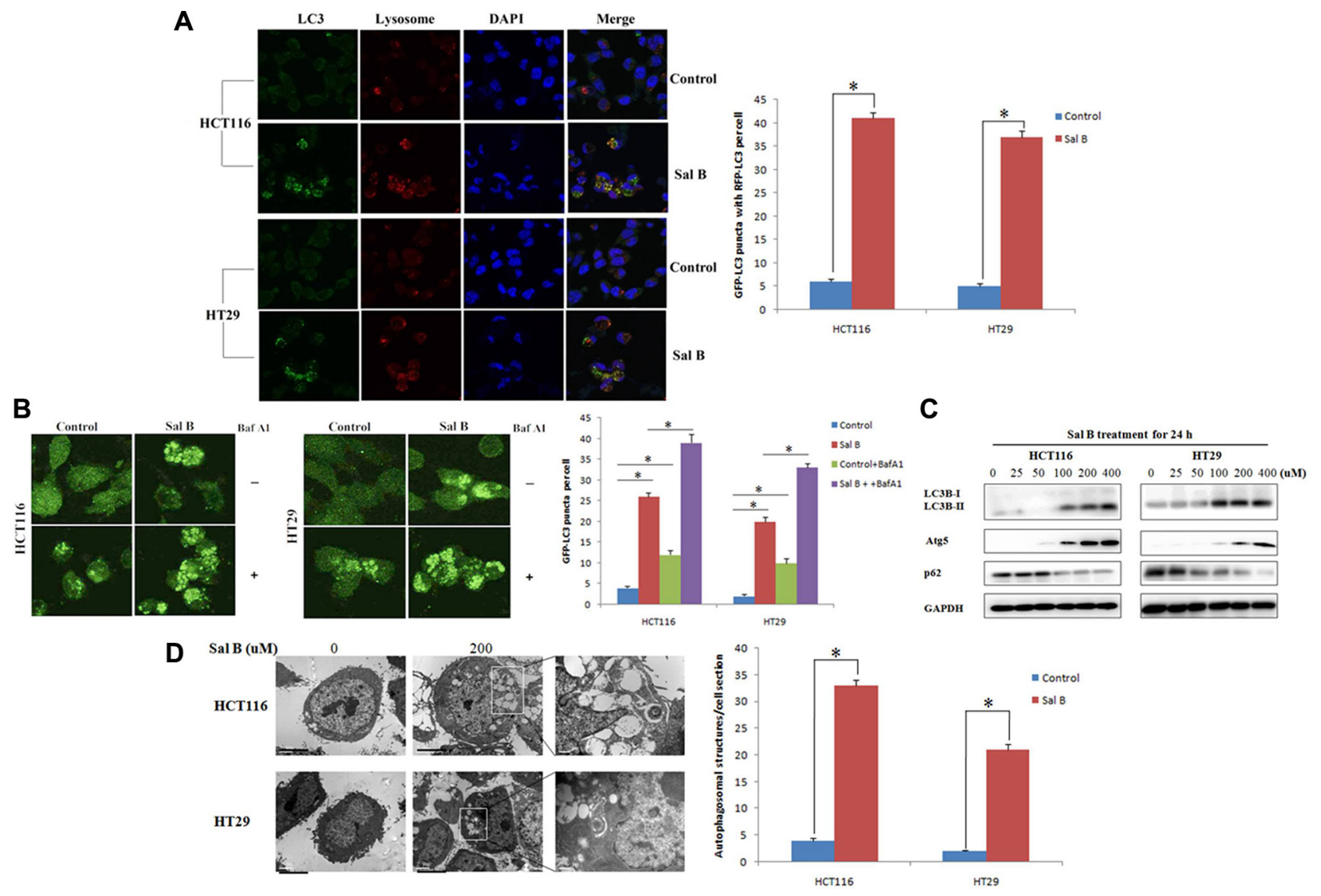

Figure 2: Aberrant autophagosome accumulation is involved in sal B-treated cells. (A) Cells were treated with Sal B for $24 \mathrm{~h}$ before they were labeled with a fluorescent marker and imaged by fluorescence microscopy. Green: FITC-labeled LC3; Red: lysotracker-labeled lysosome; Blue: DAPI-labeled nucleus. ${ }^{*} p<0.05$. (B) GFP-LC3 stable cell lines were made and Sal B-induced autophagic flux was detected in the presence and absence of BafA1 (C) The conversion from LC3-I to LC3-II as well as Atg 5 and p62, was detected by western blotting. (D) Electron microscopy shows ultrastructures of autophagosome in these cells. The experiments were performed in triplicate. $\mathrm{Bar}=1 \mu \mathrm{m} .{ }^{*} p<0.05$. 
3-MA significantly increased tumor growth compared with Sal B alone, indicating that Sal B also induced pro-death autophagy in vivo. Furthermore, no significant weight loss or hepatic toxicity was observed in the group of Sal B or a combination of 3-MA and Sal B (Figure did not show).

In order to investigate the mechanism underlying the inhibition of tumor growth by Sal B in vivo, we detected the expression of LC3B, p-AKT and p-mTOR by western blotting. As shown in Figure $\mathrm{C}$, Sal B treatment resulted in an increase in LC3-II expression. Moreover, consistent with the in vitro results, Sal B induced autophagy through the inhibition of expression of p-AKT and p-mTOR (Figure 6C).

Taken together, our data suggest that Sal B inhibited tumor growth in vivo by inducing autophagy through suppressing AKT/mTOR pathway. Inhibition of autophagy by 3-MA potently attenuated the antitumor activity of Sal B in vivo.

\section{DISCUSSION}

It is increasingly appreciated that targeting autophagy may be a promising new strategy for cancer drug discovery $[20,21]$. Moreover, the natural herbal products that can inhibit or induce autophagy are attracting more and more attention in developing novel chemotherapeutics for cancers [22-24]. In the present study, we show that Sal B is a novel autophagy inducer and exerts its antitumor activity as a single agent in colorectal cancer cells. In the further study, we found that induction of autophagy may be a key mediator for Sal B-triggered cell death and inhibition of autophagy counteracted the cytotoxic effect of Sal B in HCT116 and HT29 cells. Finally, it was clearly suggested that Sal B induced autophagic cell death in CRC cell lines by the inhibition of AKT/mTOR signaling pathway.

Autophagy is regarded as a potential target for anticancer therapy and exerts complicated functions in different stages of cancer $[25,26]$. Currently, many autophagy regulators have been identified as potential cancer therapeutic agents in pre-clinical and clinical studies [27]. Up till now, many natural products are also reported to trigger cancer cell death by regulating the signal pathways or functional status of autophagy [28, 29]. Sal B is a leading bioactive component of Danshen and has been widely used for thousands of years in oriental medicine to treat cardiovascular and cerebrovascular diseases [14]. Although previous study has shown that Sal B-induced autophagy may have the protective or lethal role in starving cardiac cells [30], autophagic cell death mediated by Sal B has never been recognized. In our study, Sal B

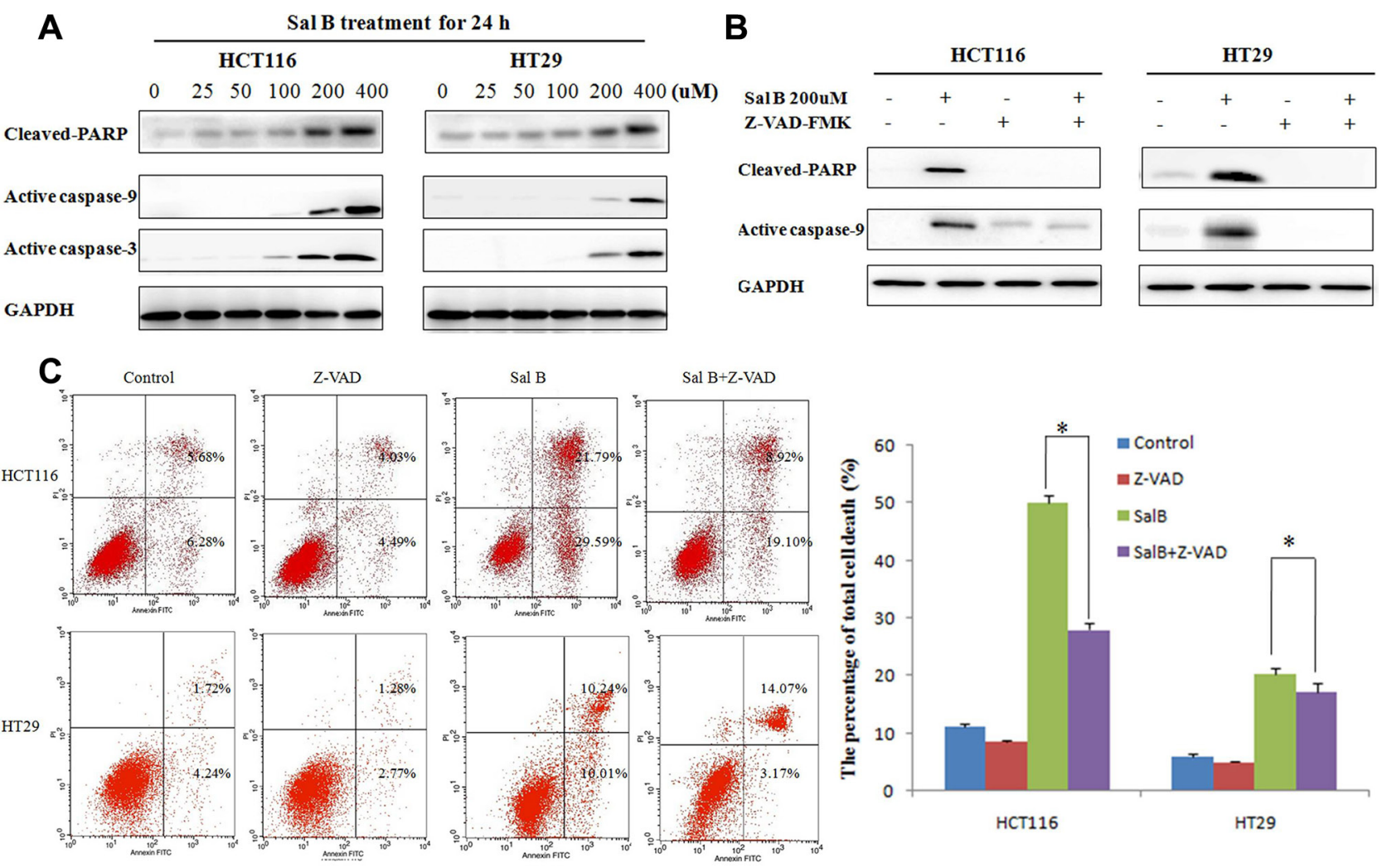

Figure 3: Sal B-induced cell death is partially apoptotic. (A) The expression of apoptosis-associated protein cleaved PARP, active caspase-9 and caspase-3 in cells treated with Sal B for $24 \mathrm{~h}$. (B) The expression of cleaved PARP and active caspase- 9 were detected by western blotting after the cells were treated with Sal B in the presence or absence of Z-VAD-FMK. (C) Flow cytometric analysis of cell death. The data are shown as the mean $\pm \mathrm{SD}$. The experiments were performed in triplicate. ${ }^{*} p<0.05$. 
was found to induce cell death and trigger autophagy in HCT116 and HT29 cells in a dose-dependent manner. We further examined the role of autophagy in Sal B-induced cell death via pretreatment with autophagy inhibitor or the silence of autophagy associated gene Atg5 with shRNA. Our results show that Sal B-induced cell death was rescued by autophagy inhibition, suggesting that Sal B-induced autophagy may play a pro-death role and contribute to the cell death of CRC cell lines.
AKT/mTOR signaling pathway has emerged as the central conduit in the regulation of autophagy. Accumulating evidence has highlighted that the inhibition of AKT and its downstream target mTOR and p70S6K contribute to the initiation of autophagy [31, 32]. Our results show that Sal B treatment exerts a inhibitory effect on AKT activation and the phosphorylation of its downstream targets, including mTOR and p70S6K. Overexpression of AKT by the transfection with pcDNA3-CA-AKT plasmid
A

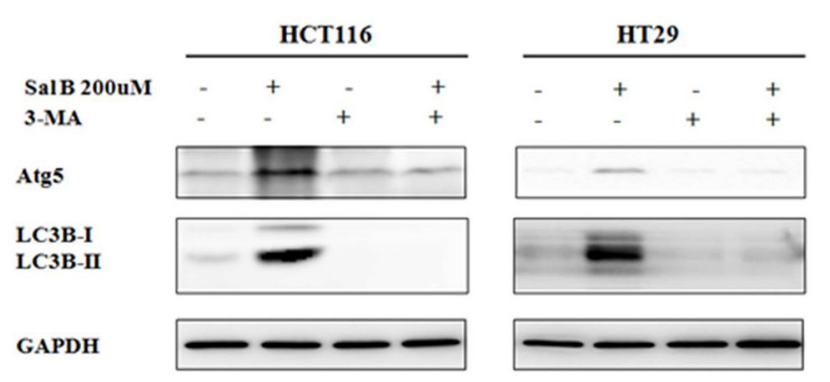

C

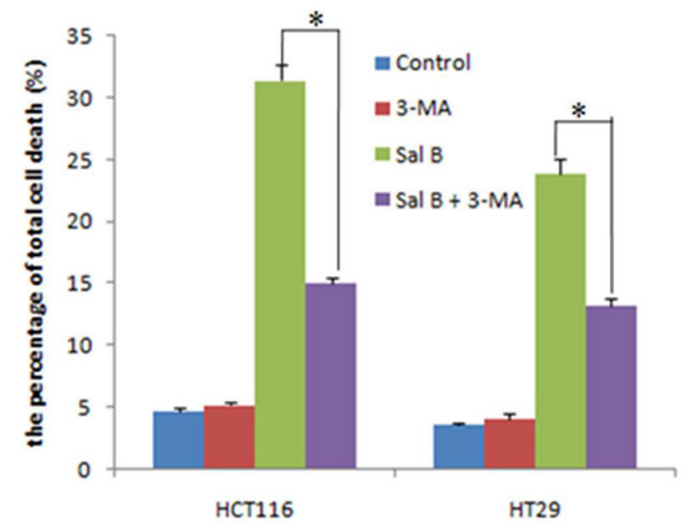

E

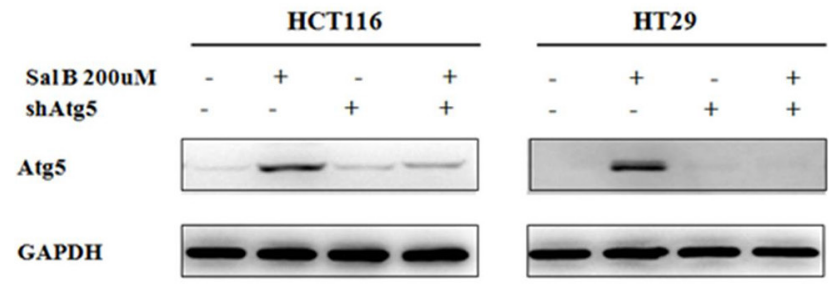

B
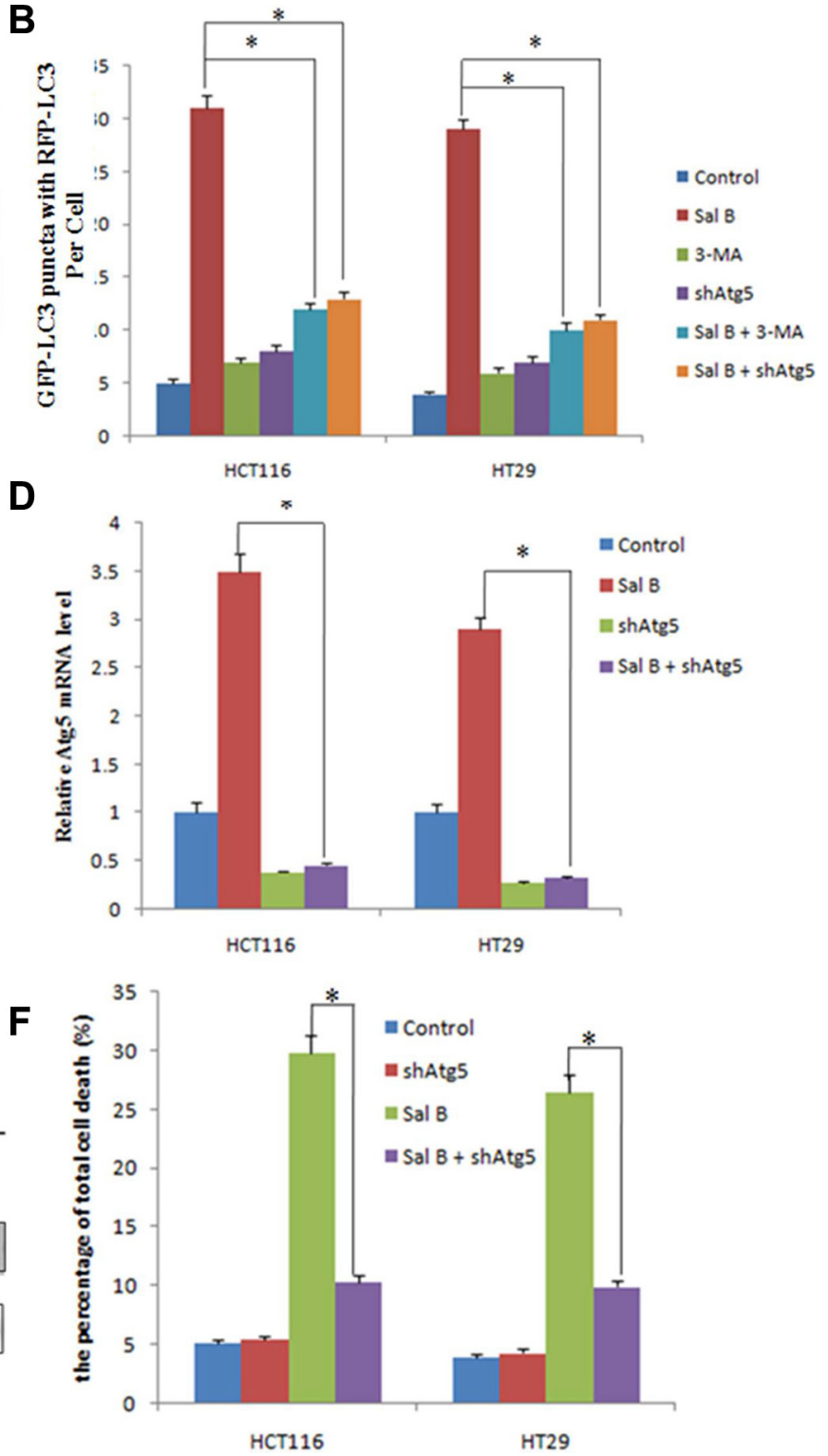

Figure 4: Autophagy contributes to sal B-induced cell death. (A) The expression of Atg5 and LC3-II/I ratio were detected by western blotting after the cells were treated with Sal B in the presence or absence of 3-MA. (B) Cells were treated with Sal B in the presence or absence of 3-MA for $24 \mathrm{~h}$ before they were labeled with a fluorescent marker and imaged by fluorescence microscopy. Green: FITClabeled LC3; Red: lyso-tracker-labeled lysosome; Blue: DAPI-labeled nucleus. ${ }^{*} p<0.05$. (C) Flow cytometric analysis of cell death. The data are shown as the mean $\pm \mathrm{SD}$. The experiments were performed in triplicate. ${ }^{*} p<0.05$. (D) The Atg5 mRNA level was detected by RT-PCR after the cells were treated with Sal B in the presence or absence of shAtg5. (E) The protein expression of Atg5 was detected by western blotting after the cells were treated with Sal B in the presence or absence of shAtg5. (F) Flow cytometric analysis of cell death. The data are shown as the mean $\pm \mathrm{SD}$. The experiments were performed in triplicate. ${ }^{*} p<0.05$. 
or pretreatment with insulin decreased Sal B-induced autophagy and cell death. Inversely, PI3K/AKT/mTOR signaling pathway inhibitor LY294002 treatment markedly enhanced Sal B-induced autophagy and cell death. Thus, AKT/mTOR signaling pathway is a critical mediator in regulating Sal B-induced cell death.

In conclusion, our present data show, for the first time, that Sal B is a novel autophagy inducer and exerts its antitumor activity as a single agent in colorectal cancer cells, both in vitro and in vivo, through the suppression of AKT/mTOR pathway. Our findings have important clinical implications. Next, we will compare the cytotoxicity of Sal $B$ with or without combination of several anticancer drugs (such as 5-FU, Oxaliplatin and Irinotecan) in preclinical models and investigate the effect of Sal B on the invasion and metastasis of CRC cells.
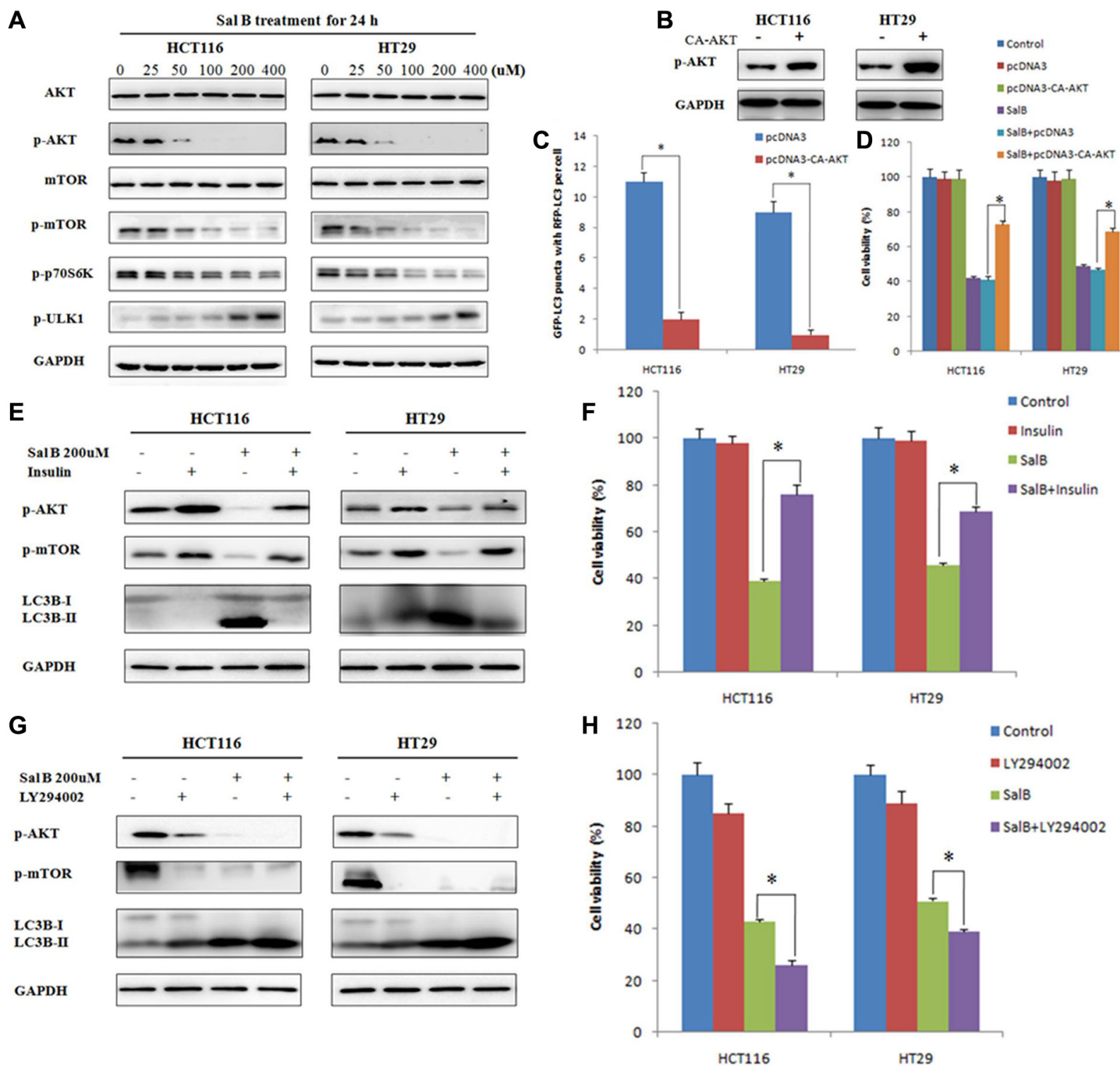

Figure 5: AKT pathway is a critical mediator in regulating sal B-induced cell death. (A) The proteins associated Akt/mTOR axis were analyzed by western blotting after the cells were treated with Sal B. (B) HCT116 and HT29 cells were transiently transfected with pcDNA3-CA-AKT plasmid. (C) Cells were treated with Sal B in the presence or absence of pcDNA3-CA-AKT plasmid for $24 \mathrm{~h}$ before they were labeled with a fluorescent marker and imaged by fluorescence microscopy. Green: FITC-labeled LC3; Red: lyso-tracker-labeled lysosome; Blue: DAPI-labeled nucleus. ${ }^{*} p<0.05$. (D) After transient overexpression of constitutively active AKT, cells were treated with Sal B for $24 \mathrm{~h}$, and then cell viability was determined by MTT. (E) After pretreatment with insulin for 30 min, cells were treated with Sal $\mathrm{B}$ for $24 \mathrm{~h}$, and then the expression of p-AKT, p-mTOR and LC3-II/I ratio were detected by western blotting. (F) After pretreatment with insulin for $30 \mathrm{~min}$, cells were treated with Sal B for $24 \mathrm{~h}$, and then cell viability was determined by MTT. * $p<0.05$. (G) After pretreatment with LY294002, cells were treated with Sal B for $24 \mathrm{~h}$, and then the expression of p-AKT, p-mTOR and LC3-II/I ratio were detected by western blotting. (H) After pretreatment with LY294002, cells were treated with Sal B for 24 h, and then cell viability was determined by MTT. $* p<0.05$. 


\section{MATERIALS AND METHODS}

\section{Reagents and antibodies}

Sal B was purchased from the National Institute for the Control of Pharmaceutical and Biological Products (Beijing, China). BafA1 (B1793), 3-methyladenine (M9281), pan caspase inhibitor Z-VADFMK (C2105) were purchased from Sigma Aldrich. Proteins were reacted with one of the following: anti-LC-3B (\#3868), anti-p62 (\#8025), anti-Atg5 (\#2630), anti-PARP (\#9532), anticleaved PARP (\#5625), anti-cleaved caspase-3 (\#9661), anti-cleaved caspase-9 (\#7237), anti-AKT (\#9272), antiphospho-AKT (Ser473) (\#4060), anti-mTOR (\#2972), anti-phospho-mTOR (Ser2448) (\#5536), anti-phosphop70S6K (Thr389) (\#9234), anti- phospho-ULK1 (Ser757) (\#6888) were purchased from Cell Signaling Technology.

\section{Cell lines and animals}

Human colon cancer cell lines HCT116 and HT29 were obtained from American Type Culture Collection. HCT116 cells were maintained in RPMI 1640 medium (Invitrogen, 11875-093). HT29 cells were maintained in McCoy's 5A (Invitrogen, 16600-082). All media were supplemented with $10 \%(\mathrm{v} / \mathrm{v})$ fetal bovine serum

(FBS), $1 \%(\mathrm{v} / \mathrm{v})$ of $100 \mathrm{U} / \mathrm{ml}$ penicillin and $100 \mathrm{mg} /$ mlstreptomycin. Cells were cultured at $37^{\circ} \mathrm{C}$ in a humidified 5\% $\mathrm{CO}_{2}$ incubator. Sal $\mathrm{B}$ was dissolved in double-distilled $\mathrm{H}_{2} \mathrm{O}$ and was further diluted with medium before use. Female athymic BALB/c nude mice (Shanghai Institute of Material Medicine, Chinese Academy of Science, China) were maintained in a specific pathogenfree facility and were treated with humane care after approval from the Animal Care and Use Committee of Zhejiang University.

\section{Measurement of cell viability and apoptosis}

Cell viability was determined by MTT assay. Cells were seeded in 96-well flat bottom microtiter plates at a density of $1 \times 10^{4}$ cells per well. $24 \mathrm{~h}$ later, Sal B was added at the concentrations indicated for $24 \mathrm{~h}$. The absorbance was measured on a microplate reader (Synergy HT, Bio-Tek, USA) at $570 \mathrm{~nm}$. Phartmingen annexin V-FITC Apoptosis Ddtection Kit I (BD, USA) was used to detect apoptosis and the estimation procedure was performed according to the manufacturer's instructions. $2 \times 10^{6}$ cells were seeded into a $6 \mathrm{~cm}$ dish. After attachment overnight, cells were washed twice with PBS and the medium was replaced medium with Sal B for $24 \mathrm{~h}$. All cells including the floating cells in the culture medium
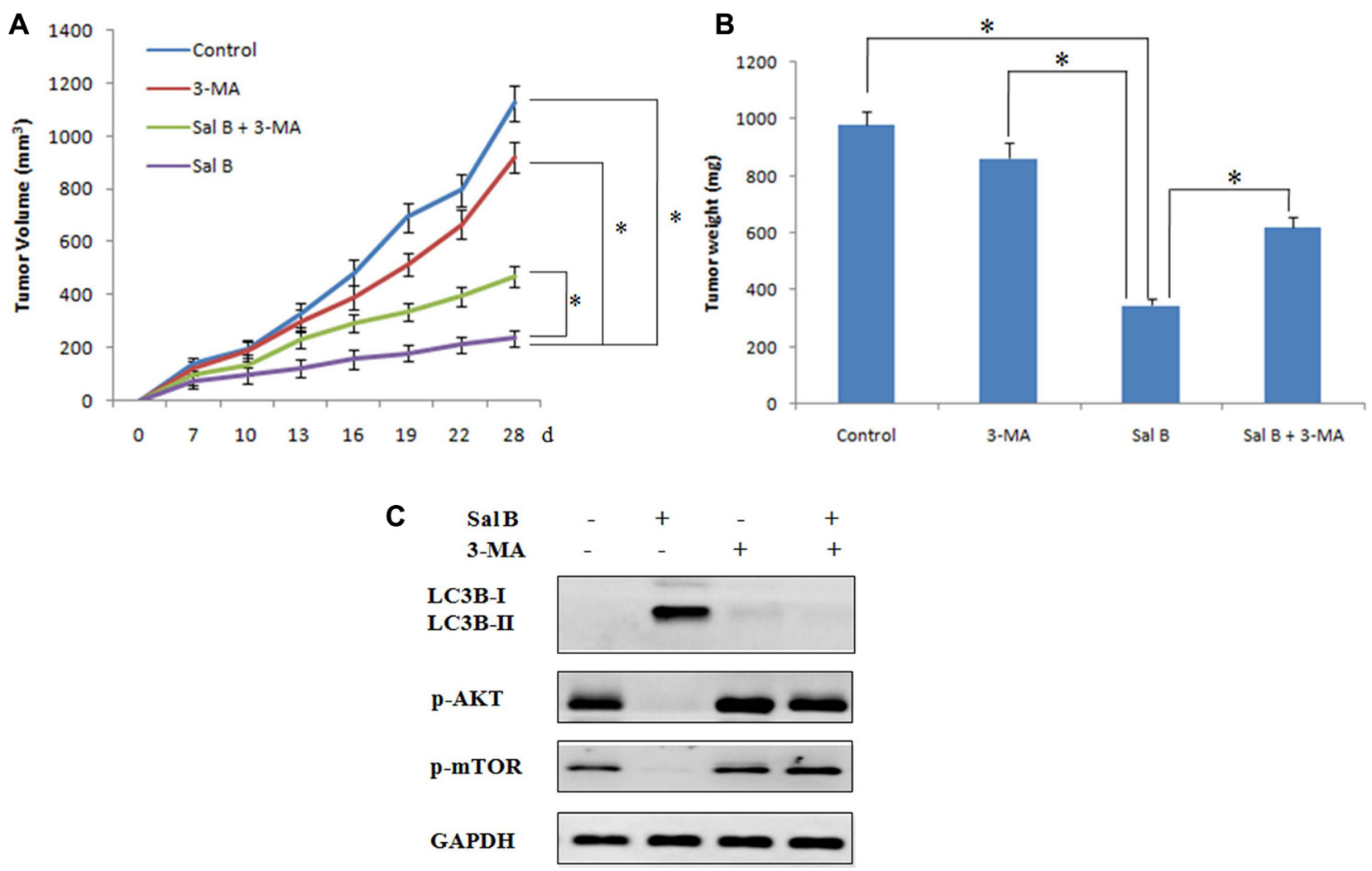

Figure 6: Sal B exerts autophagy induction and antitumor efficacy in HCT116 xenograft models. (A) Tumor volume in each group. Data are expressed as the mean \pm SEM. (B) Tumor weight in each group. Data were expressed as the mean $\pm \mathrm{SD},{ }^{*} p<0.05$. (C) Western blotting analysis of protein expression in tumors taken from mice. 
were harvested. The cells were resuspended in ice-cold $1 \times$ binding buffer at a concentration of $1 \times 10^{6}$ cells $/ \mathrm{ml}$. $100 \mu \mathrm{l}$ of cell suspension were each mixed with $5 \mu \mathrm{l}$ FITC Annexin V and $5 \mu \mathrm{l}$ PI. The mixture was incubated for $15 \mathrm{~min}$ at room temperature in the dark and then analyzed by FACSCalibur Flow Cytometer (BD Biosystems, Heidelberg, Germany).

\section{Colony formation assays}

Cells were trypsinized and plated in triplicate into $10 \mathrm{~cm}$ dishes at different densities based on cell types. Cells were treated with the indicated concentrations of Sal B or vehicle control for $24 \mathrm{~h}$. Twenty-four hours after Sal B treatment, the medium was removed and cells were maintained in normal culturing medium. Two weeks after the cells were plated, they were washed and stained with crystal violet, and the colonies containing $>50$ cells were counted.

\section{Semi-quantitative RT-PCR}

Total RNA was isolated using TRIzol reagent (Invitrogen, CA, USA) according to the manufacturer's instructions. Approximately $2 \mu \mathrm{g}$ of total RNA was converted to cDNA by First-strand cDNA synthesis kit (OriGene Technologies, MD, USA). Semi-quantitative RT-PCR was performed as described before. The GAPDH mRNA sequence was also amplified as an internal control.

\section{Immunofluorescent confocal laser microscopy for LC3 and lysosome co-location}

Lysosome was firstly labeled by incubation with Lyso Tracker (Invitrogen, L7528), a lysosome reporter dye, for $90 \mathrm{~min}$ at $37^{\circ} \mathrm{C}$. Cells were collected, fixed and permeabilized with $1 \%$ CHAPS buffer $(150 \mathrm{mM} \mathrm{NaCl}$, $10 \mathrm{mM}$ HEPES, $1.0 \%$ CHAPS) at room temperature for $10 \mathrm{~min}$, incubated with anti-LC3 for $2 \mathrm{~h}$ at room temperature, and washed with PBS, incubated for another $45 \mathrm{~min}$.

\section{Plasmid and RNA interference}

GPF-LC3 lentiviral plasmid (LTV-801) was purchased from Cell Biolabs, INC. Cells were transfected with either nonspecific siRNA (Qiagen,1027280) and Atg5 siRNA (Qiagen, SI02655310) via LipofectAMINE RNAi max (Invitrogen, 13778150) according to the manufacturer's instructions.

\section{Transmission electron microscopy}

Treated cells were washed and fixed for $30 \mathrm{~min}$ in $2.5 \%$ glutaraldehyde. The samples were treated with $1.5 \%$ osmium tetroxide, dehydrated with acetone and embedded in Durcupan resin. Thin sections were poststained with lead citrate andexamined in the TECNAI 10 electron microscope (Philips, Holland) at $60 \mathrm{kV}$.

\section{Western blot analysis}

Cells were harvested from cultured dishes and were lysed in a lysis buffer [20 mM Tris- $\mathrm{HCl} \mathrm{pH} 7.6,1 \mathrm{mM}$ EDTA, $140 \mathrm{mM} \mathrm{NaCl}, 1 \% \mathrm{NP}-40,1 \%$ aprotinin, $1 \mathrm{mM}$ phenylemethylsulfonyl fluoride (PMSF), $1 \mathrm{mM}$ sodium vanadate]. Protein concentration was determined using a BCA Protein Assay Kit (Pierce). Cell lysates (40 $\mu \mathrm{g}$ protein/line) were separated on a 5 to $20 \%$ Tris-Tricine Ready Gel SDS-PAGE (Bio-Rad) for nitrocellulose membrane blotting. The blotted membranes were blocked with $5 \%$ skim milk for $1 \mathrm{~h}$ and were incubated with primary antibodies. The immunoreactive bands were visualized by enhanced chemiluminescence using horseradish perox-idase-conjugated $\mathrm{IgG}$ secondary antibodies. Band density was measured by densitometry, quantified using gel plotting macros of NIH image 1.62, and normalized to an indicated sample in the identical membrane.

\section{In vivo subcutaneous tumor model}

All of the in vivo experimental protocols were approved by the animal care committee of Sir Run Run Shaw Hospital, Zhejiang University. Viable HCT116 cells $\left(1 \times 10^{7}\right.$ cells in $0.1 \mathrm{ml}$ phosphate buffer saline $)$ were injected subcutaneously into right dorsal flank of 6-weekold female BALB/c nude mice (six mice per group). Tumor volume was assessed every 2 days for 4 weeks. Tumor volume was calculated by the following formula: $(\text { short diameter })^{2} \times($ long diameter $) / 2$.

\section{Statistical analyses}

Results are expressed as values of mean \pm standard deviation (SD). Statistical analysis was performed using SPSS 16.0 for Windows (SPSS Inc., Chicago, IL, USA). The correlation coefficient of two factors was evaluated using Chi-square and Fisher's exact tests. The survival of patients with colorectal adenocarcinomas was compared using the Kaplan-Meier method, and differences between the survival curves were tested using the logrank test. A $P$-value less than 0.05 is considered significant.

\section{ACKNOWLEDGMENTS AND FUNDING}

This study is supported by grants from Zhejiang province science and technology project of TCM (grant No. 2015ZB033), National Natural Science Foundation of China (grant No. 81301891, 81572361 and 81572592) and Zhengshu Medical Elite Scholarship Fund. 


\section{CONFLICTS OF INTEREST}

The authors declare no conflicts of interest.

None of the contents of this manuscript has been previously published or is under consideration elsewhere. All the authors read and approved the final version of the manuscript prior to submission.

\section{Author's contributions}

X.S., W.H. and H.P. designed the study. X.S. wrote the main manuscript text. X.S., J.Z., W.F., L.Z., L.C., X.Z., X.L., J.X. and Y.F. performed experiments. J.Z. and W.F. prepared figures. J.Z. and L.Z. did the statistical analyses. All authors reviewed the manuscript.

\section{REFERENCES}

1. Siegel R, Ma J, Zou Z, Jemal A. Cancer statistics, 2014. CA Cancer J Clin. 2014; 64:9-29.

2. Zhang Y, Talmon G, Wang J. MicroRNA-587 antagonizes 5-FU-induced apoptosis and confers drug resistance by regulating PPP2R1B expression in colorectal cancer. Cell Death Dis. 2015; 6:e1845.

3. Chen J, Lu H, Yan D, Cui F, Wang X, Yu F, Xue Y, Feng X, Wang J, Wang X, Jiang T, Zhang M, Zhao S, et al. PAK6 increase chemoresistance and is a prognostic marker for stage II and III colon cancer patients undergoing 5-FU based chemotherapy. Oncotarget. 2015; 6:355-367. doi: 10.18632/oncotarget.2803.

4. Kondo Y, Kanzawa T, Sawaya R, Kondo S. The role of autophagy in cancer development and response to therapy. Nature reviews Cancer. 2005; 5:726-734.

5. Galluzzi L, Pietrocola F, Bravo-San Pedro JM, Amaravadi RK, Baehrecke EH, Cecconi F, Codogno P, Debnath J, Gewirtz DA, Karantza V, Kimmelman A, Kumar S, Levine B, et al. Autophagy in malignant transformation and cancer progression. EMBO J. 2015; 34:856-880.

6. Janku F, McConkey DJ, Hong DS, Kurzrock R. Autophagy as a target for anticancer therapy. Nat Rev Clin Oncol. 2011; $8: 528-539$.

7. Garcia-Cano J, Ambroise G, Pascual-Serra R, Carrion MC, Serrano-Oviedo L, Ortega-Muelas M, Cimas FJ, Sabater S, Ruiz-Hidalgo MJ, Sanchez Perez I, Mas A, Jalon FA, Vazquez A, et al. Exploiting the potential of autophagy in cisplatin therapy: A new strategy to overcome resistance. Oncotarget. 2015; 6:15551-15565. doi: 10.18632/ oncotarget. 3902 .

8. Buchser WJ, Laskow TC, Pavlik PJ, Lin HM, Lotze MT. Cell-mediated autophagy promotes cancer cell survival. Cancer Res. 2012; 72:2970-2979.

9. El Hasasna H, Athamneh K, Al Samri H, Karuvantevida N, Al Dhaheri Y, Hisaindee S, Ramadan G, Al Tamimi N, AbuQamar S, Eid A, Iratni R. Rhus coriaria induces senescence and autophagic cell death in breast cancer cells through a mechanism involving p38 and ERK1/2 activation. Sci Rep. 2015; 5:13013.

10. Zhou L, Zuo Z, Chow MS. Danshen: an overview of its chemistry, pharmacology, pharmacokinetics, and clinical use. J Clin Pharmacol. 2005; 45:1345-1359.

11. Lin F, Liu YY, Xu B, Sun K, Wang HY, Li Q, Wang CS, Fan JY, Zhang SW, Han JY. Salvianolic acid B protects from pulmonary microcirculation disturbance induced by lipopolysaccharide in rat. Shock. 2013; 39:317-325.

12. Chen T, Liu W, Chao X, Zhang L, Qu Y, Huo J, Fei Z. Salvianolic acid B attenuates brain damage and inflammation after traumatic brain injury in mice. Brain research bulletin. 2011; 84:163-168.

13. Ren Z, Wang X, Wang S, Zhai C, He Y, Zhang Y, Qiao Y. Mechanism of action of salvianolic acid $\mathrm{B}$ by module-based network analysis. Bio-medical materials and engineering. 2014; 24:1333-1340.

14. Wang ZS, Luo P, Dai SH, Liu ZB, Zheng XR, Chen T. Salvianolic acid B induces apoptosis in human glioma U87 cells through p38-mediated ROS generation. Cellular and molecular neurobiology. 2013; 33:921-928.

15. Wang QL, Wu Q, Tao YY, Liu CH, El-Nezami H. Salvianolic acid B modulates the expression of drugmetabolizing enzymes in HepG2 cells. Hepatobiliary \& pancreatic diseases international. 2011; 10:502-508.

16. Yang Y, Ge PJ, Jiang L, Li FL, Zhu QY. Modulation of growth and angiogenic potential of oral squamous carcinoma cells in vitro using salvianolic acid B. BMC complementary and alternative medicine. 2011; 11:54.

17. Zhao Y, Guo Y, Gu X. Salvianolic Acid B, a potential chemopreventive agent, for head and neck squamous cell cancer. Journal of oncology. 2011; 2011:534548.

18. Shi J, Wang H, Guan H, Shi S, Li Y, Wu X, Li N, Yang C, Bai X, Cai W, Yang F, Wang X, Su L, et al. IL10 inhibits starvation-induced autophagy in hypertrophic scar fibroblasts via cross talk between the IL10-IL10R-STAT3 and IL10-AKT-mTOR pathways. Cell Death Dis. 2016; 7:e2133.

19. Yang Z, Klionsky DJ. Eaten alive: a history of macroautophagy. Nat Cell Biol. 2010; 12:814-822.

20. Lebovitz CB, DeVorkin L, Bosc D, Rothe K, Singh J, Bally M, Jiang X, Young RN, Lum JJ, Gorski SM. Precision autophagy: Will the next wave of selective autophagy markers and specific autophagy inhibitors feed clinical pipelines? Autophagy. 2015; 11:1949-1952.

21. Shao FY, Du ZY, Ma DL, Chen WB, Fu WY, Ruan BB, Rui W, Zhang JX, Wang S, Wong NS, Xiao H, Li MM, Liu X, et al. B5, a thioredoxin reductase inhibitor, induces apoptosis in human cervical cancer cells by suppressing the thioredoxin system, disrupting mitochondrion-dependent pathways and triggering autophagy. Oncotarget. 2015; 6:30939-30956. doi: 10.18632/oncotarget.5132.

22. Wang X, Qi W, Li Y, Zhang N, Dong L, Sun M, Cun J, Zhang Y, Lv S, Yang Q. Huaier Extract Induces Autophagic 
Cell Death by Inhibiting the mTOR/S6K Pathway in Breast Cancer Cells. PloS one. 2015; 10:e0131771.

23. Zhao X, Fang Y, Yang Y, Qin Y, Wu P, Wang T, Lai H, Meng L, Wang D, Zheng Z, Lu X, Zhang H, Gao Q, et al. Elaiophylin, a novel autophagy inhibitor, exerts antitumor activity as a single agent in ovarian cancer cells. Autophagy. 2015; 11:1849-1863.

24. Zhao F, Huang W, Zhang Z, Mao L, Han Y, Yan J, Lei M. Triptolide induces protective autophagy through activation of the CaMKKbeta-AMPK signaling pathway in prostate cancer cells. Oncotarget. 2016; 7:5366-5382. doi: 10.18632/oncotarget.6783.

25. Pandey S, Chandravati. Autophagy in cervical cancer: an emerging therapeutic target. Asian Pacific journal of cancer prevention. 2012; 13:4867-4871.

26. Shintani T, Klionsky DJ. Autophagy in health and disease: a double-edged sword. Science. 2004; 306:990-995.

27. Sui X, Chen R, Wang Z, Huang Z, Kong N, Zhang M, Han W, Lou F, Yang J, Zhang Q, Wang X, He C, Pan H. Autophagy and chemotherapy resistance: a promising therapeutic target for cancer treatment. Cell Death Dis. 2013; 4:e838.
28. Zhong W, Zhu H, Sheng F, Tian Y, Zhou J, Chen Y, Li S, Lin J. Activation of the MAPK11/12/13/14 (p38 MAPK) pathway regulates the transcription of autophagy genes in response to oxidative stress induced by a novel copper complex in HeLa cells. Autophagy. 2014; 10:1285-1300.

29. Lao Y, Wan G, Liu Z, Wang X, Ruan P, Xu W, Xu D, Xie W, Zhang Y, Xu H, Xu N. The natural compound oblongifolin $\mathrm{C}$ inhibits autophagic flux and enhances antitumor efficacy of nutrient deprivation. Autophagy. 2014; 10:736-749.

30. Han X, Liu JX, Li XZ. Salvianolic acid B inhibits autophagy and protects starving cardiac myocytes. Acta Pharmacol Sin. 2011; 32:38-44.

31. Huang C, Lin MZ, Cheng D, Braet F, Pollock CA, Chen XM. KCa3.1 mediates dysfunction of tubular autophagy in diabetic kidneys via PI3k/Akt/mTOR signaling pathways. Sci Rep. 2016; 6:23884.

32. Lu Q, Yan S, Sun H, Wang W, Li Y, Yang X, Jiang X, Che Y, $\mathrm{Xi}$ Z. Akt inhibition attenuates rasfonin-induced autophagy and apoptosis through the glycolytic pathway in renal cancer cells. Cell Death Dis. 2015; 6:e2005. 